\title{
Manufacture of primary mirror segments for the Giant Magellan Telescope
}

H. M. Martin, R. Allen, V. Gasho, B. T. Jannuzi, D. W. Kim, et al.

H. M. Martin, R. Allen, V. Gasho, B. T. Jannuzi, D. W. Kim, J. S. Kingsley, K. Law, A. Loeff, R. D. Lutz, T. J. McMahon, C. J. Oh, M. T. Tuell, S. N. Weinberger, S. C. West, "Manufacture of primary mirror segments for the Giant Magellan Telescope," Proc. SPIE 10706, Advances in Optical and Mechanical Technologies for Telescopes and Instrumentation III, 107060V (10 July 2018); doi: 10.1117/12.2312935

Event: SPIE Astronomical Telescopes + Instrumentation, 2018, Austin, Texas, United States 


\title{
Manufacture of primary mirror segments for the Giant Magellan Telescope
}

\author{
H. M. Martin ${ }^{\mathrm{a}}$, R. Allen , V. Gasho ${ }^{\mathrm{a}}$, B. T. Januzzi ${ }^{\mathrm{a}}$, D. W. Kim ${ }^{\mathrm{b}}$, J. S. Kingsley ${ }^{\mathrm{a}}$, K. Law ${ }^{\mathrm{a}}$, A. Loeff \\ R. D. Lutz ${ }^{\mathrm{a}}$, T. J. McMahon ${ }^{\mathrm{a}}$, C. J. Oh ${ }^{\mathrm{b}}$, M. T. Tuell ${ }^{\mathrm{a}}$, S. N. Weinberger ${ }^{\mathrm{a}}$ and S. C. West ${ }^{\mathrm{a}}$ \\ ${ }^{a}$ Steward Observatory, University of Arizona, Tucson, AZ 85721, USA \\ ${ }^{\mathrm{b}}$ College of Optical Sciences, University of Arizona, Tucson, AZ 85721, USA
}

\begin{abstract}
The Richard F. Caris Mirror Lab at the University of Arizona continues the production of $8.4 \mathrm{~m}$ lightweight honeycomb segments for the primary mirror of the Giant Magellan Telescope. GMT will have a center segment surrounded by six identical off-axis segments, plus an additional off-axis segment to allow continuous operation as segments are removed for coating. Production highlights of the last two years include the spin-casting of Segment 5, preliminary polishing of Segment 2, and completion of the rear surface processing for Segments 3 and 4. We completed a preliminary design of the significant modifications of the test systems required for Segment 4, the center segment. We finished an upgrade of the $8.4 \mathrm{~m}$ polishing machine; both the upgrade and experience gained with Segment 1 have contributed to much faster polishing convergence for Segment 2. Prior to polishing Segment 2, we verified the stability and accuracy of the measurement systems by re-measuring Segment 1, achieving good agreement among multiple independent tests as well as good agreement with the original acceptance tests of Segment 1.
\end{abstract}

Keywords: Giant Magellan Telescope, telescopes, optical fabrication, optical testing, off-axis, aspheres

\section{INTRODUCTION}

The $25 \mathrm{~m}$ GMT primary mirror consists of seven $8.4 \mathrm{~m}$ lightweight honeycomb mirrors. ${ }^{[1]-[3]}$ The honeycomb design provides the stiffest and lightest large mirrors ever made, minimizing deflection due to gravity and wind. The use of $8.4 \mathrm{~m}$ segments guarantees a smooth wavefront over large fractions of the aperture and, together with the segmented adaptive secondary mirror, simplifies alignment and phasing of the telescope. The primary mirror segments are being made at the Richard F. Caris Mirror Lab at the University of Arizona. The first off-axis segment is finished and Segments 2-5 are in different stages of manufacture. (Segments are numbered in order of casting; Segment 4 is the center segment.)

The off-axis segments present significant challenges in fabrication and optical testing. The interferometric test has a 3element off-axis null corrector that includes a $3.75 \mathrm{~m}$ curved mirror and has overall dimensions exceeding $8 \mathrm{~m} \cdot{ }^{[4],[5]}$ To mitigate the risk of a mistake in the interferometric test, we have independent measurements of critical parameters including radius of curvature, off-axis geometry and low-order aberrations (astigmatism, coma, trefoil and spherical aberration). These tests are housed in a $28 \mathrm{~m}$ test tower.

Completion of Segment 1 demonstrated the essential technology for manufacture of all the segments, but we need to achieve significant improvements in production schedule. We made a number of improvements in methods and equipment for fabrication and testing during and after the manufacture of Segment $1 .{ }^{[6]}$ While some of the equipment upgrades delayed the start of work on the optical surface of Segment 2, so far they appear to be paying off in much more efficient figuring of Segment 2. We are confident that these improvements, and others that we are making based on the experience with Segment 2, will continue to accelerate production of the remaining segments.

\section{STATUS OF SEGMENT PRODUCTION}

GMT Segment 1, the first off-axis segment, was completed in August 2012. ${ }^{[7],[8]}$ The surface is accurate to $18 \mathrm{~nm} \mathrm{rms}$ after a simulated active-optics correction using 27 bending modes with $21 \mathrm{~N} \mathrm{rms}$ correction force. The off-axis geometry meets its tolerances, with the error in off-axis distance $=0.2 \pm 1.2 \mathrm{~mm}$ and the clocking error $=0 \pm 13$ arcseconds. The clocking reference is defined by the loadspreaders bonded to the back of the mirror.

Advances in Optical and Mechanical Technologies for Telescopes and Instrumentation III, edited by Ramón Navarro, Roland Geyl, Proc. of SPIE Vol. 10706, 107060V · (C) 2018 SPIE CCC code: $0277-786 \mathrm{X} / 18 / \$ 18 \cdot$ doi: $10.1117 / 12.2312935$ 
Segments 2 and 3 are also off-axis segments. Segment 2 was cast in January 2012 and is currently being polished. The long interval between casting and polishing included fabrication of the $8.4 \mathrm{~m}$ combined primary and tertiary mirrors for the Large Synoptic Survey Telescope (LSST) ${ }^{[9]}$, generating or milling the $6.5 \mathrm{~m}$ primary mirror for the Tokyo Atacama Observatory, and major upgrades to both of our $8.4 \mathrm{~m}$ machines, the Large Optical Generator (LOG) and the Large Polishing Machine (LPM). Segment 3 was cast in August 2013. We have completed work on the rear surface, including bonding the 165 loadspreaders that form the interface between the mirror and its support system. Segment 3 is currently moving onto the LOG for generating of the optical surface.

Segment 4, the center segment, was cast in September 2015. We recently finished grinding and polishing the rear surface and we are currently bonding the loadspreaders. Segment 5 was cast in November 2017. We are currently removing the ceramic fiber mold parts that produce the honeycomb structure and remain inside the mirror until they are washed out with high-pressure water. Figure 1 shows Segment 5 shortly after the furnace was opened, following the spin-casting and a 90-day cooling period. We have purchased 40 tons of Ohara Corporation's E6 borosilicate glass for Segments 6 and 7.

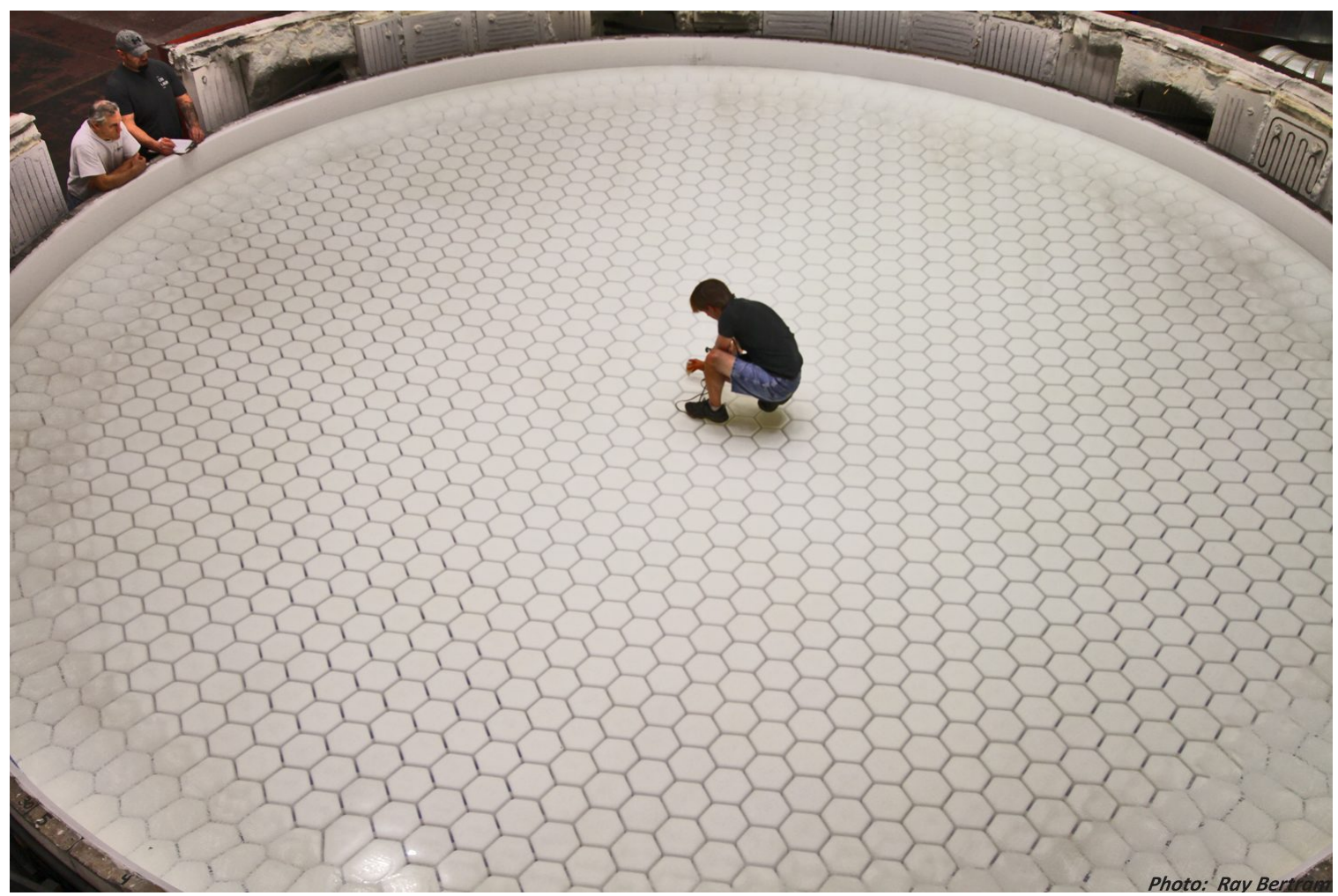

Figure 1. Senior Staff Technician Damon Jackson uses an ultrasonic gauge to measure the thickness of the facesheet after the spin-casting of GMT Segment 5. Photo by Ray Bertram, University of Arizona.

\section{FABRICATION OF SEGMENT 2}

\subsection{Polishing system and initial results}

We started polishing Segment 2 in January 2018 and are still in the early stages. Most of the polishing has been done with an orbital polishing system using a variety of relatively small tools that give good spatial resolution for controlling the figure ${ }^{[10]}$ Figure 2 shows the polishing process in February 2018.

The surface accuracy is $340 \mathrm{~nm} \mathrm{rms}$ at the time of writing, an improvement by almost two orders of magnitude from the generated surface, with just over another order of magnitude needed before it will meet its figure specification. Figure 3 shows the figure convergence through the first 2-1/2 months of figuring, starting with the first optical test (the SCOTS test described in Section 3.2). For comparison with Segment 1, we show its convergence through about the same range of rms surface error, from $2400 \mathrm{~nm}$ to $320 \mathrm{~nm}$. 




Figure 2. GMT Segment 2 being polished with a $40 \mathrm{~cm}$ orbital lap on the Large Polishing Machine.

Through the range shown, progress is about $4 \times$ faster for Segment 2. We don't assume this rate of convergence will continue to completion, but we are confident that the rate will continue to be substantially better than we achieved throughout the fabrication of Segment 1, and that the process improvements we have made and continue to make will produce segments at a rate consistent with the telescope schedule.

Through the early stages of polishing, we evaluate the rms surface error from SCOTS $^{[11],[12]}$ after subtracting focus, astigmatism, coma and trefoil, a conservative approximation to the residual error after an active-optics correction using segment displacement and bending ${ }^{[8]}$ SCOTS has high accuracy for structure with periods of 2-3 $\mathrm{m}$ and smaller, roughly the range of scales that are not corrected with active optics, but it does not measure low-order aberrations accurately. The interferometric measurement (principal test, also described in Section 3.2) measures all spatial scales accurately enough to meet requirements, but in the early stages its coverage of the mirror is limited by high slope errors. Starting at about the current figure accuracy, the principal test and SCOTS together fully define the surface on all scales down to about a $20 \mathrm{~mm}$ period. Going forward, we will simulate the active-optics correction rather than approximating it by subtracting Zernike polynomials.

For Segment 1, we did not have optical test data until day 85 on the graph in Figure 3, we did not implement the SCOTS test until day 198, and we needed several additional months to refine both the SCOTS test and methods of making use of SCOTS data. The most important process improvement for Segment 2 was to have a mature SCOTS test available from the time the surface was polished and shiny enough to measure with visible light. Additional factors that contribute to the improved convergence include a new orbital polishing head with improved stiffness, speed, torque and reliability; a new and more reliable control system for the LPM; a transition from 24 hours $\times 5$ days to $24 \times 7$ operation; a more efficient data processing pipeline; and more experience using the computer-controlled polishing system with dwell varying as a function of position on the mirror. 


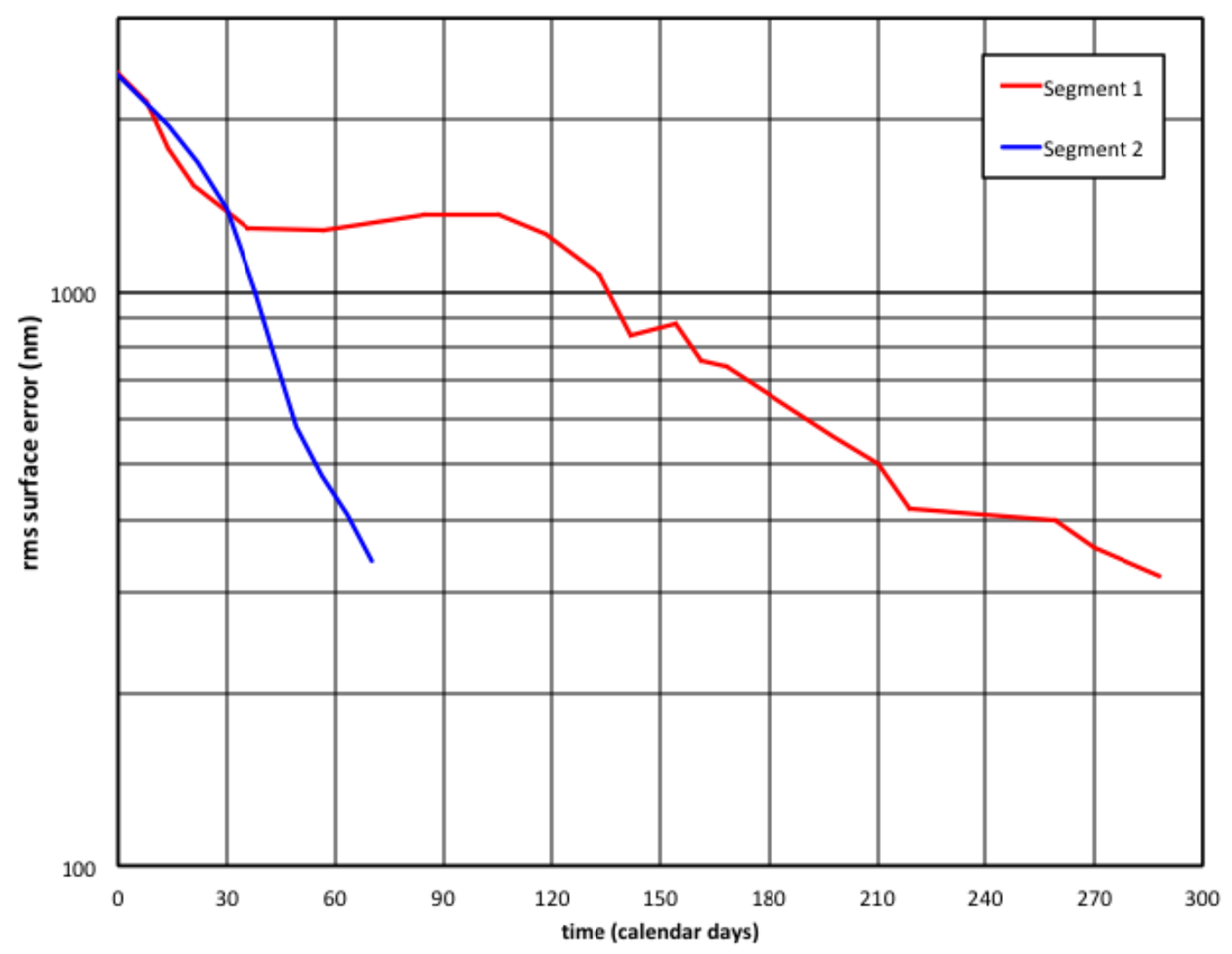

Figure 3. Convergence chart showing rms surface error vs time for GMT Segments 1 and 2. The range of rms surface error covers the work to date for Segment 2, starting with the first optical test. The rms surface error is evaluated after subtracting focus, astigmatism, coma and trefoil, an approximation to the residual after an active-optics correction.

\subsection{Validation of test systems}

Prior to polishing and measuring GMT Segment 2, we verified the accuracy of the test systems by re-measuring Segment 1. In the interval since we last measured Segment 1 in 2012, we made several upgrades to the test systems, including both hardware and data-processing software. We used Segment 1 as a reference to verify that these upgrades did not cause any changes that conflict with previous results, and that no unexpected changes occurred during the roughly 5 years since we used the tests.

The test systems are large and complex, and require alignment to a demanding accuracy. Sensitivities to alignment errors are much greater for the off-axis GMT segments than for comparable axisymmetric mirrors. In addition, in order to form a single $25 \mathrm{~m}$ near-parabolic primary mirror, the segments require essentially identical radii of curvature after an activeoptics correction. The limited range of active correction then implies that each segment's radius must be controlled to about $0.5 \mathrm{~mm}$, just over one part in $10^{4}$, during manufacture. While we can show on paper that each test meets its accuracy requirements, the only way to have sufficient confidence in the accuracy of the finished mirror is to have independent measurements of all critical parameters and to require agreement among multiple tests. These independent tests are listed below.

- The interferometric test, or principal test, measures the full aperture on all spatial scales down to its roughly $10 \mathrm{~mm}$ sampling, as well as radius of curvature. ${ }^{[4],[5]}$ It measures all spatial scales to accuracies that meet the segment specifications, as long as the slope errors are within the capture range of the interferometer. It uses a large and complex null corrector-comprising a $3.75 \mathrm{~m}$ spherical mirror, a $0.76 \mathrm{~m}$ sphere, and a computer-generated hologram - to shape the test wavefront to match the ideal off-axis segment with its $13 \mathrm{~mm} \mathrm{p}$-v aspheric departure. 
- The Software Configurable Optical Test System (SCOTS) is a deflectometry system that measures slopes over the full aperture with high spatial resolution and dynamic range. ${ }^{[11],[12]}$ The slopes are integrated to give a full surface measurement with high accuracy for periods up to 2-3 m.

- The scanning pentaprism test is the main independent verification of off-axis geometry and large-scale figure. ${ }^{[13]-[15]}$ It synthesizes a star test by illuminating the segment with a narrow collimated beam parallel to the telescope's optical axis and scanning the beam across the segment in multiple cuts. The beam comes to a focus on a detector at the telescope's prime focus, where the spot motion is proportional to the slope error on the segment. (There is a small spot motion in the ideal case because the primary mirror is not parabolic, with $k=-0.9983$ ). We fit a model to the slope data to determine the radius of curvature, astigmatism, coma, trefoil and spherical aberration.

- The Laser Tracker Plus (LT+) system is a scan of the full surface with a laser tracker, with stability references to eliminate the effects of rigid-body motion. ${ }^{[16],[17]} \mathrm{LT}+$ works with a ground or polished surface. It is accurate to about $1 \mu \mathrm{m}$ rms surface and $0.4 \mathrm{~mm}$ in radius of curvature.

The final measurements of Segment 1 in 2012 showed agreement among the tests, within the expected uncertainties, for radius of curvature, off-axis distance, clocking, and low-order aberrations through spherical. ${ }^{[7]}$ These are the parameters that are sensitive to alignment errors in the tests and, therefore, the parameters most vulnerable to a mistake in any one test. The agreement among the tests guarantees that the mirror surface can be corrected with active optics in the telescope to eliminate low-order errors and leave only mid-scale and small-scale errors of about $20 \mathrm{~nm}$ rms.

The main goal of the new measurements of Segment 1 in 2017 was to obtain the same results we obtained during the acceptance tests in 2012, within the uncertainties of each test. We also expected to obtain agreement for low-order aberrations among all tests performed in 2017. Finally, we expected the best estimates of radius of curvature (a weighted average of LT+, the pentaprism test and the principal test) in 2012 and 2017 to agree within the 2- $\sigma$ uncertainty of 0.4 $\mathrm{mm}$. We quoted 2- $\sigma$ uncertainties for all measured parameters in 2012 and we used the same uncertainties to set criteria for successful results of the reference tests.

With one slight exception discussed below, the 2017 results meet all the goals listed above. Figure 4 shows one important example, the error in radius of curvature measured with all three tests in 2012 and 2017. All data are in agreement. The extreme consistency of results in 2017 is probably a coincidence and is not typical of results for other parameters. The weighted average of the three measurements is also in good agreement, with $\Delta R=0.12 \pm 0.4 \mathrm{~mm}$ in 2012 and $-0.04 \pm 0.4 \mathrm{~mm}$ in 2017.

The one, marginal exception to excellent agreement is the $30^{\circ}$ component of trefoil measured with the principal test. In 2012 we estimated a $2-\sigma$ uncertainty of $40 \mathrm{~nm}$ for the difference of two measurements. (The polynomial is normalized to unit rms, so the coefficient equals the rms surface error.) The 2012 and 2017 measurements differ by $41 \mathrm{~nm}$. More importantly, the variation among multiple measurements in 2017 is larger than expected, indicating that we underestimated one or more sources of non-repeating error. We have not yet identified the additional error, but it has almost no impact on performance of the mirror in the telescope because trefoil is easily corrected with active optics. The variation among measurements indicates that we need to increase the $2-\sigma$ uncertainty of a single measurement to $60 \mathrm{~nm}$, and average at least 3 measurements for final acceptance tests. We have shown that the effect of this additional uncertainty after the active-optics correction is $<1 \mathrm{~N}$ increase in rms correction force and $<1 \mathrm{~nm}$ increase in rms residual error after correction, well within the margins available.

The agreement between results in 2012 and 2017, and the agreement among the three tests, gives high confidence that the tests are sufficiently stable and accurate to demonstrate that the GMT segments will match within tolerances and will meet all requirements at the telescope. The final acceptance tests of each segment are required to show agreement among the three tests. Segment 4, the center segment, requires major modifications to the tests, described in Section 4.2. When the tests are later re-configured for off-axis Segment 5, we plan to validate the tests by re-measuring one of the finished off-axis segments. 


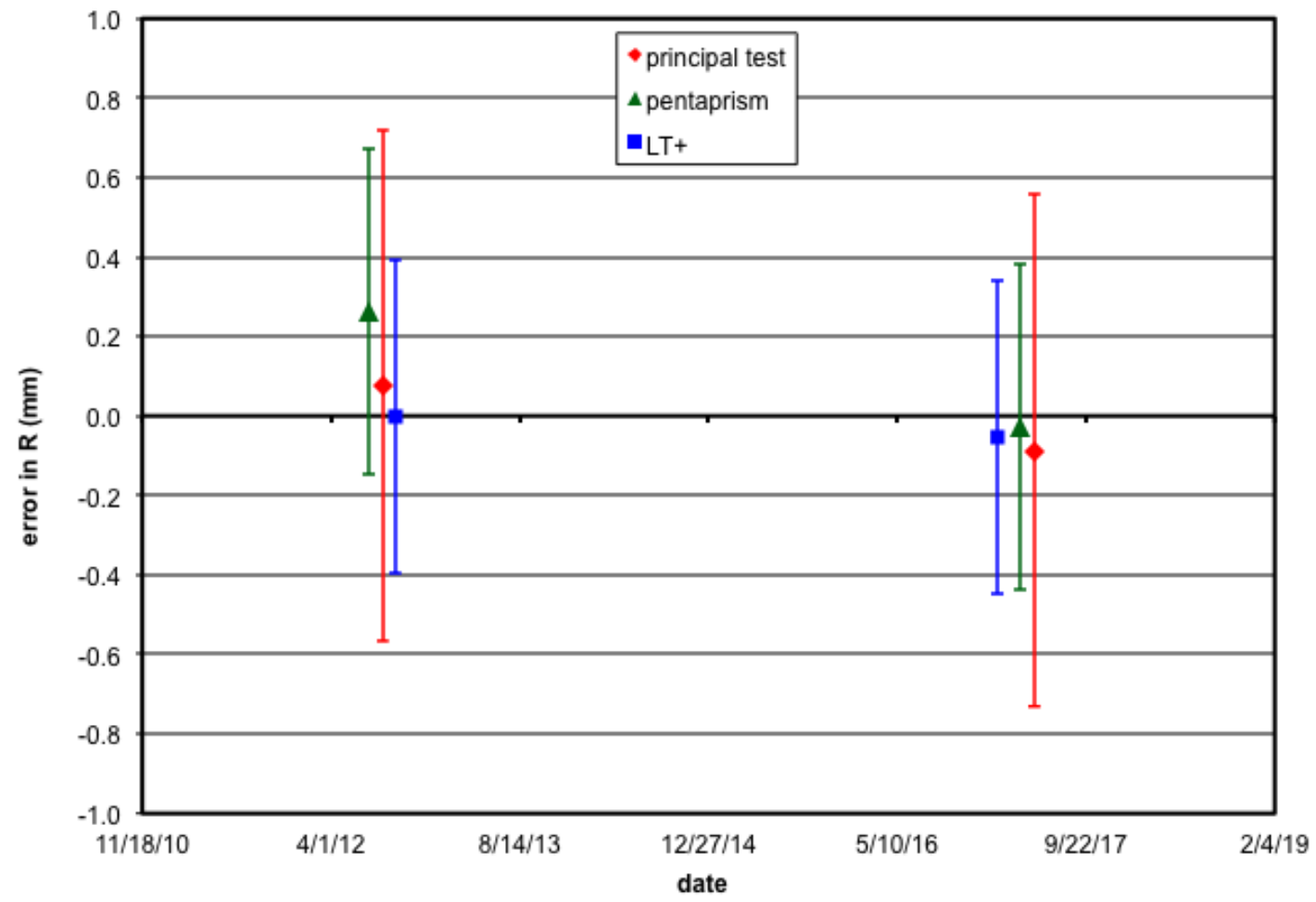

Figure 4. Measurements of Segment 1's radius of curvature with three independent tests, in 2012 and 2017. The nominal radius of the parent is $36 \mathrm{~m}$. Error bars are our estimates of $2-\sigma$ uncertainties. For all 3 tests the error in $R$ comes from a combination of distance and Zernike focus aberration.

\section{PROGRESS ON SEGMENTS 3 AND 4}

\subsection{Fabrication status}

We have completed work on the rear surfaces of Segment 3 and 4, including generating and polishing. (We polish the rear surface to facilitate visual inspection of the internal honeycomb structure and maximize strength against impact.) We have bonded loadspreaders to the back of Segment 3 and are in the process of bonding them to Segment 4 . Figure 5 shows Segment 3 with loadspreaders attached. Segment 3 has since been turned right-side up in preparation for generating the optical surface to an accuracy of about $20 \mu \mathrm{m}$ rms.

\subsection{Optical tests for the center segment}

Manufacture of Segment 4, the center segment, requires significant modifications to the optical tests. The Mirror Lab recently completed a preliminary design of the full test plan for Segment 4, and passed a design review in March 2018.

We will use the same tests listed in Section 3.2. Of the four tests, LT + is unchanged but the three others will move to be positioned symmetrically over the mirror. As shown in Figure 6, the principal test and SCOTS will still use the $3.75 \mathrm{~m}$ spherical mirror to make a folded path, but the fold mirror is moved to nadir-pointing. We studied the possibility of keeping the same asymmetric geometry used for the off-axis segments - and modifying the null corrector to compensate for the severe asymmetry of the test-but rejected it because of a number of difficulties in creating an accurate symmetric test wavefront. 


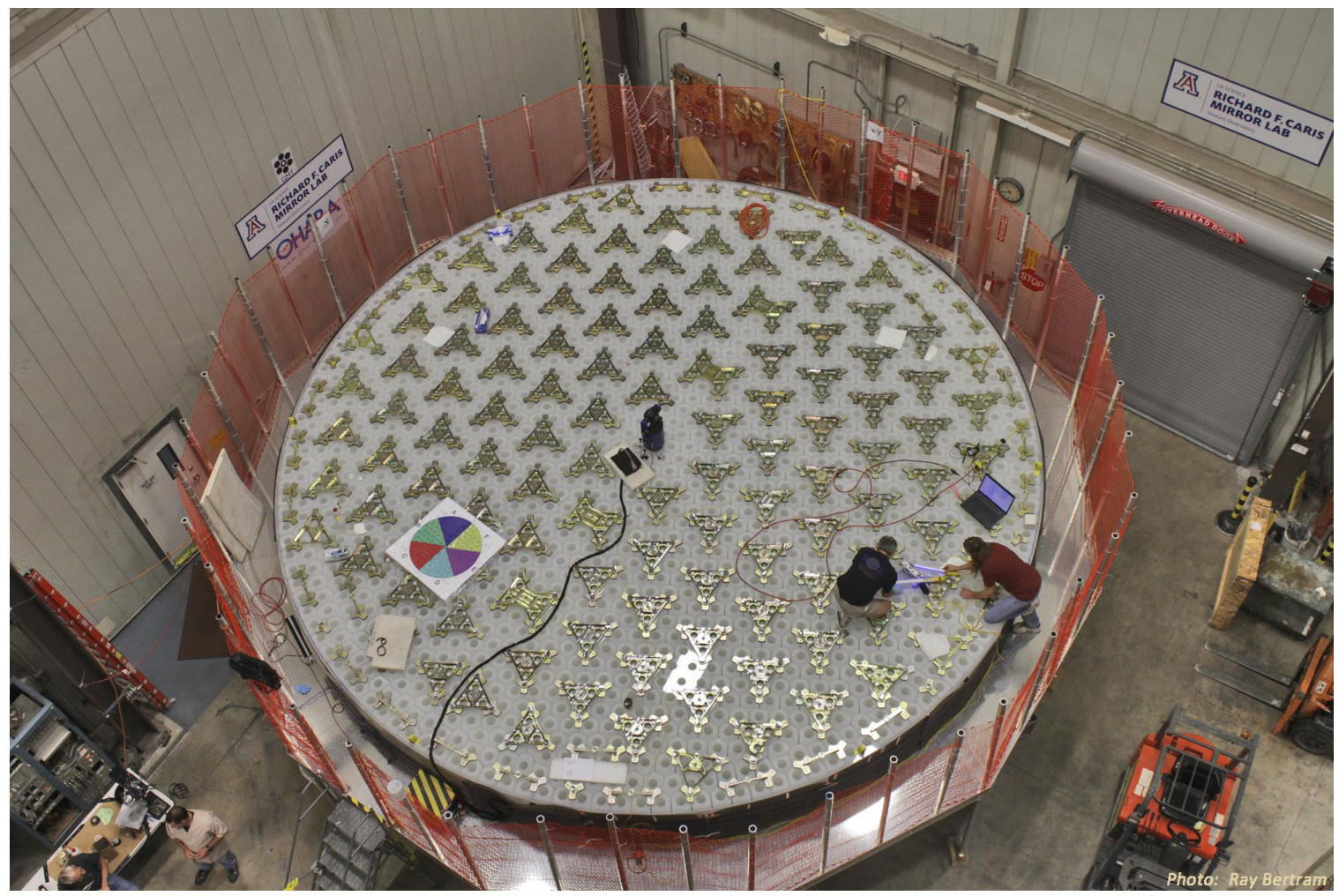

Figure 5. Overhead view of the back of GMT Segment 3 during a final verification of position accuracy for the 165 loadspreaders that form the interface between the mirror and its support system. The loadspreaders are positioned to $0.5 \mathrm{~mm}$ using the laser tracker seen at the center of the mirror. Photo by Ray Bertram, University of Arizona.

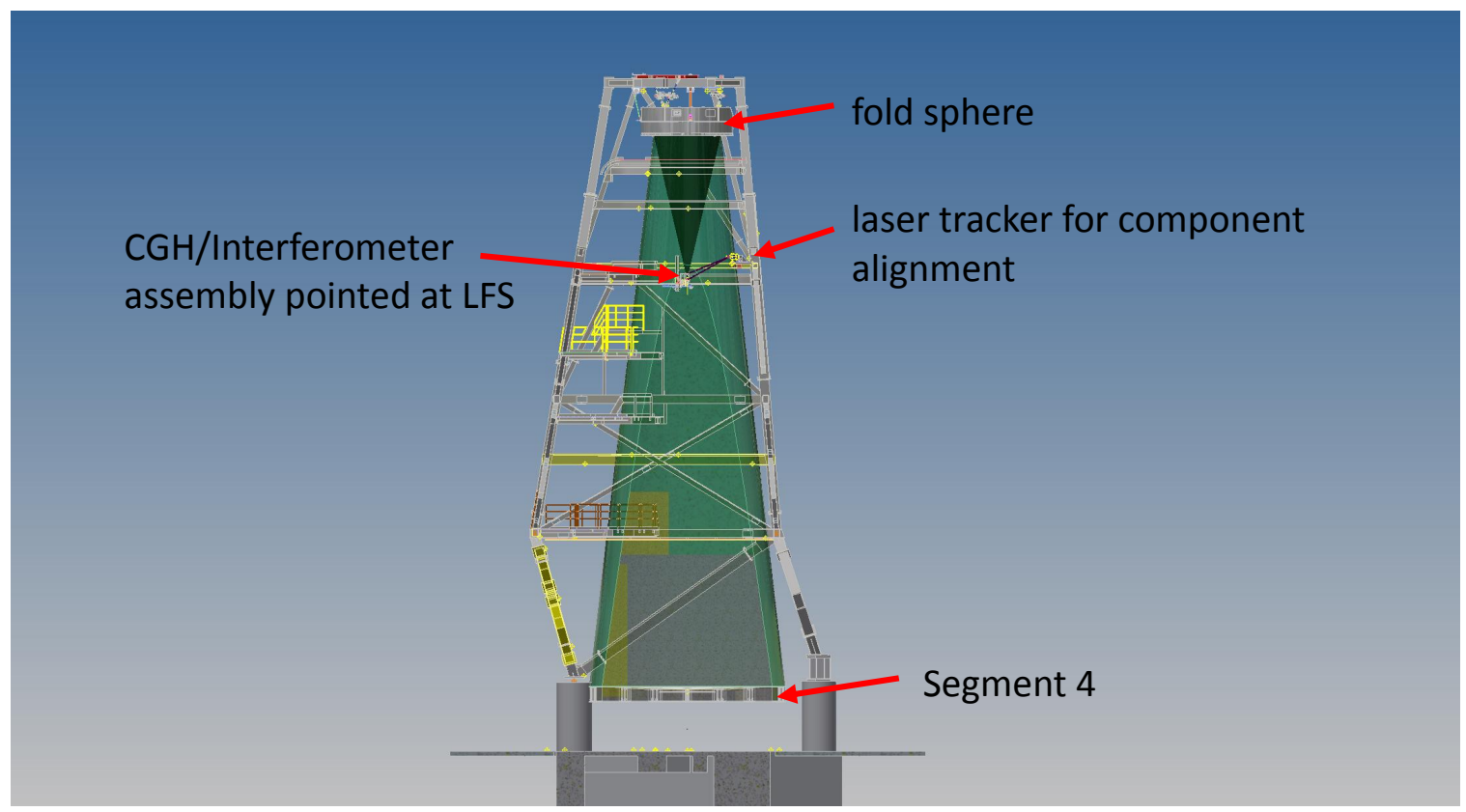

Figure 6. Layout of the principal test for GMT Segment 4, the center segment, in the $28 \mathrm{~m}$ test tower. The principal test and SCOTS use a folded path going through a $3.75 \mathrm{~m}$ spherical mirror at the top of the tower. The interferometer and CGH (first element of the null corrector) are centered above Segment 4. 
Unlike the off-axis test, the folded path for the principal test and SCOTS causes a modest obscuration. We can pack the test elements (interferometer and CGH for the principal test, projecting screen and camera for SCOTS) into a volume that projects onto the $2.4 \mathrm{~m}$ Cassegrain hole in Segment 4, but their supporting structure blocks the beam in narrow strips totaling $3 \%$ of the measured aperture. We minimize the impact of obscurations by mounting all the test elements on deployable bridges so, for example, the interferometer and SCOTS systems do not obscure the pentaprism test, and vice versa. We can also clock Segment 4 by $90^{\circ}$ to make every part of the aperture visible in one orientation or the other. We faced a similar challenge in simultaneous measurement of LSST's primary and tertiary mirrors - the test bridge for the tertiary obscured part of the primary - and we were able to obtain sufficient data to demonstrate compliance with all requirements.

The components of the pentaprism test also move to be centered over the mirror. The pentaprism rail must be perpendicular to the parent's optical axis, so it is moved into a horizontal plane, and the pentaprism detector moves to the new prime focus position $18 \mathrm{~m}$ directly above Segment 4 . With all components deployable, there is no significant obscuration in the pentaprism test.

\section{CASTING OF SEGMENT 5}

The spin-casting of Segment 5 took place in November 2017, with the glass melting into the honeycomb mold on November 4. The casting went smoothly through 3 months of cooling, and the furnace was opened on February 6, 2018 to reveal another high-quality GMT mirror blank. It was lifted off the furnace hearth and moved to the handling ring, where it could be tilted to a vertical plane for removal of the mold pieces that are trapped in the mirror during the casting. Figure 7 shows the mirror prepared for the lift off of the hearth.

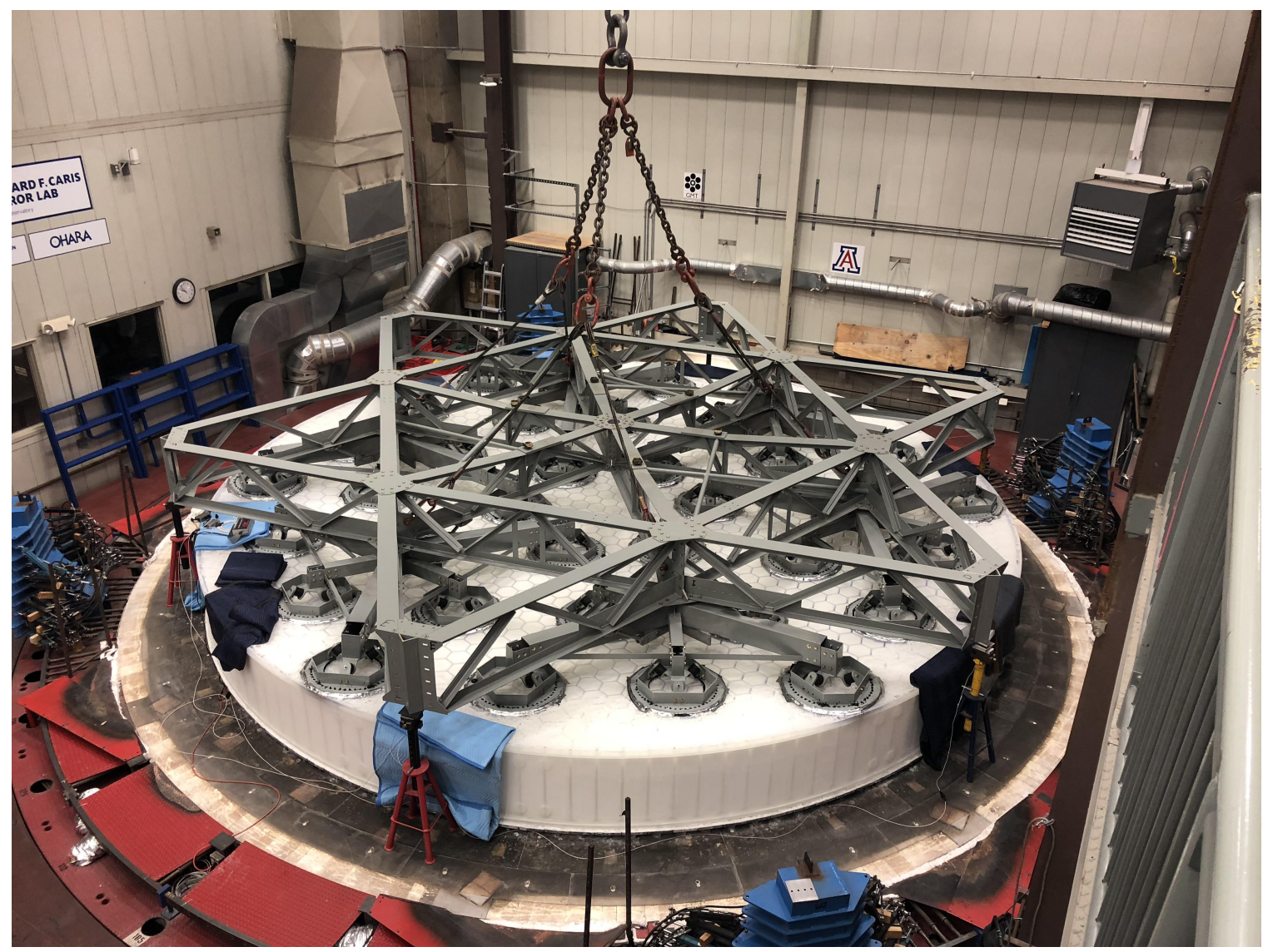

Figure 7. GMT Segment 5 prepared for the lift off of the furnace hearth following the spin-casting. The lifting fixture is bonded to the front surface with compliant adhesive applied to 36 disks. 
The ceramic fiber mold material is washed out of the mirror with high-pressure water. We have access to the internal surfaces through a $90 \mathrm{~mm}$ hole in the backplate centered on each of the 1681 hexagonal cavities. (The same holes are used to ventilate the mirror in operation.) The clean-out is nearly complete at the time of writing. Preliminary inspections show the mirror to be in excellent shape with no significant flaws. A complete inspection will be carried out after the mirror has thoroughly dried.

\section{CONCLUSION}

The Mirror Lab continues a vigorous program to produce primary mirror segments for GMT. Four segments are in various stages of manufacture following the completion of Segment 1 . We recently celebrated the successful casting of Segment 5. Recent upgrades to the generating and polishing systems are, to date, paying off in improved polishing convergence for Segment 2. These upgrades and experience gained with Segments 1 and 2 will improve the production rate for the remaining GMT segments.

\section{ACKNOWLEDGMENT}

This work has been supported by the GMTO Corporation, a non-profit organization operated on behalf of an international consortium of universities and institutions: Arizona State University, Astronomy Australia Ltd, the Australian National University, the Carnegie Institution for Science, Harvard University, the Korea Astronomy and Space Science Institute, the São Paulo Research Foundation, the Smithsonian Institution, the University of Texas at Austin, Texas A\&M University, the University of Arizona, and the University of Chicago.

\section{REFERENCES}

[1] Patrick J. McCarthy, James Fanson, Rebecca Bernstein, David Ashby, Bruce Bigelow, Nune Boyadjian, Antonin Bouchez, Eric Chauvin, Eduardo Donoso, Jose Filgueira, Robert Goodrich, Frank Groark, George Jacoby, Eric Pearce, "Overview and status of the Giant Magellan Telescope Project," Proc. SPIE 9906, Ground-based and Airborne Telescopes VI, 990612 (8 August 2016); doi: 10.1117/12.2234505.

[2] R. A. Bernstein, P. J. McCarthy, K. Raybould, B. C. Bigelow, A. H. Bouchez, J. M. Filgueira, G. H. Jacoby, M. Johns, D. Sawyer, S. Shectman and M. Sheehan, "Overview and status of the Giant Magellan Telescope project", Proc. SPIE 9145, Ground-based and Airborne Telescopes V, 91451C (2014).

[3] M. Johns, C. Hull, G. Muller, B. Irarrazaval, A. Bouchez, T. Chylek, C. Smith, A. Wadhavkar, B. Bigelow, S. Gunnels, B. McLeod and C. Buleri, "Design of the Giant Magellan Telescope", Proc. SPIE 9145, Ground-based and Airborne Telescopes V, 91451F (2014).

[4] J. H. Burge, W. Davison, H. M. Martin and C. Zhao, "Development of surface metrology for the Giant Magellan Telescope primary mirror", Proc. SPIE 7018, Advanced Optical and Mechanical Technologies in Telescopes and Instrumentation, 701814 (2008).

[5] S. C. West, J. H. Burge, B. Cuerden, W. B. Davison, J. Hagen, H. M. Martin, M. T. Tuell and C. Zhao, "Alignment and use of the optical test for the $8.4 \mathrm{~m}$ off-axis primary mirrors of the Giant Magellan Telescope", Proc. SPIE 7739, Modern Technologies in Space- and Ground-based Telescopes and Instrumentation, 77390N (2010).

[6] H. M. Martin, J. H. Burge, J. M. Davis, D. W. Kim, J. S. Kingsley, K. Law, A. Loeff, R. D. Lutz, C. Merrill, P. A. Strittmatter, M. T. Tuell, S. N. Weinberger, S. C. West, "Status of mirror segment production for the Giant Magellan Telescope," Proc. SPIE 9912, Advances in Optical and Mechanical Technologies for Telescopes and Instrumentation II, 99120V (22 July 2016); doi: 10.1117/12.2234491.

[7] H. M. Martin, R. G. Allen, J. H. Burge, J. M. Davis, W. B. Davison, M. Johns, D. W. Kim, J. S. Kingsley, K. Law, R. D. Lutz, P. A. Strittmatter, P. Su, M. T. Tuell, S. C. West and P. Zhou, "Production of primary mirror segments for the Giant Magellan Telescope", Proc. SPIE 9151, Advances in Optical and Mechanical Technologies for Telescopes and Instrumentation, 91510J (2014).

[8] H. M. Martin, R. G. Allen, J. H. Burge, D. W. Kim, J. S. Kingsley, K. Law, R. D. Lutz, P. A. Strittmatter, P. Su, M. T. Tuell, S. C. West and P. Zhou, "Production of $8.4 \mathrm{~m}$ segments for the Giant Magellan Telescope", Proc. SPIE 8450, Modern Technologies in Space- and Ground-based Telescopes and Instrumentation II, 84502D (2012).

[9] H. M. Martin, R. G. Allen, J. H. Burge, B. Cuerden, W. J. Gressler, W. Hubler, D. W. Kim, J. S. Kingsley, K. Law, P. A. Strittmatter, M. T. Tuell, S. C. West, C. Zhao and P. Zhou, "Manufacture of the combined primary and tertiary mirrors of the Large Synoptic Survey Telescope", Proc. SPIE 9151, Advances in Optical and Mechanical Technologies for Telescopes and Instrumentation, 915125 (2014). 
${ }^{[10]}$ Dae Wook Kim, James H. Burge, Jonathan M. Davis, Hubert M. Martin, Michael T. Tuell, Logan R. Graves, Steve C. West, "New and improved technology for manufacture of GMT primary mirror segments," Proc. SPIE 9912, Advances in Optical and Mechanical Technologies for Telescopes and Instrumentation II, 99120P (22 July 2016); doi: $10.1117 / 12.2231911$.

[11] P. Su, R. E. Parks, L. Wang, R. Angel and J. H. Burge, "Software configurable optical test system: a computerized reverse Hartmann test," Applied Optics, Vol 49, 4404-4412 (2010).

[12] P. Su, S. Wang, M. Khreishi, Y. Wang, T. Su, R. E. Parks, P. Zhou, M. Rascon, T. Zobrist, H. Martin and J. H. Burge, "SCOTS: A reverse Hartmann test with high dynamic range for Giant Magellan Telescope primary mirror segments", Proc. SPIE 8450, Modern Technologies in Space- and Ground-based Telescopes and Instrumentation II, 84500W (2012).

[13] P. Su, J. H. Burge, B. Cuerden and H. M. Martin, "Scanning pentaprism measurements of off-axis aspherics", Proc. SPIE 7018, Advanced Optical and Mechanical Technologies in Telescopes and Instrumentation, 70183T (2008).

[14] P. Su, J. H. Burge, B. Cuerden, R. G. Allen and H. M. Martin, "Scanning pentaprism measurements of off-axis aspherics II," Proc. SPIE 7426, Optical Manufacturing and Testing VIII, 74260Y (2009).

[15] R. G. Allen, J. H. Burge, P. Su and H. M. Martin, "Scanning pentaprism test for the GMT $8.4 \mathrm{~m}$ off-axis segments", Proc. SPIE 7739, Modern Technologies in Space- and Ground-based Telescopes and Instrumentation, 773911 (2010).

[16] T. Zobrist, J. H. Burge, W. Davison and H. M. Martin, "Measurement of large optical surfaces with a laser tracker", Proc. SPIE 7018, Advanced Optical and Mechanical Technologies in Telescopes and Instrumentation, 70183U (2008).

[17] T. L. Zobrist, J. H. Burge and H. M. Martin, "Accuracy of laser tracker measurements of the GMT 8.4 m off-axis mirror segments", Proc. SPIE 7739, Modern Technologies in Space- and Ground-based Telescopes and Instrumentation, 77390S (2010). 